\title{
Investigation of the relationship between groups and subgroups in C3S's structure transition
}

\section{Dounia Tlamsamani, Mbark Ait Mouha, Khalid Yamni}

Materials, Molecular Engineering Environment Laboratory, Moulay Ismail University, Zitoune 11201, Morocco;

douniayns@gmail.com

In recent years, many studies have been carried out on cement and its phases to understand the morphology, and to control the mineralogy of this material; due to the great position it has become globally occupied. This material is formed from a synthetic rock called clinker; tricalcium silicate $(\mathrm{Ca} 3 \mathrm{SiO} 5$ or $\mathrm{C} 3 \mathrm{~S})$ its major constituent present a concentration from $40 \%$ to $70 \%$, and its solid solution with various impurities is called alite. Impure $\mathrm{C} 3 \mathrm{~S}$ exhibits seven polymorphs from ambient temperature to $1500^{\circ} \mathrm{C}$; three forms triclinic (T1, T2, T3), three monoclinic (M1, M2, M3) and one shape rhombohedral (R). At room temperature, impurities stabilize some of the high temperature forms of the pure compound. Those forms are related by transformation matrix determined in this article. The aim of the present paper is to investigate the structural modulations of alite.

Keywords: cement; polymorph; crystal structure; alite 